\title{
Automatical object identification based on Hu moments
}

\author{
Hongrui Lin ${ }^{1}$, Xiaoguo Zhang ${ }^{2, a}$ and Gang Chen ${ }^{1}$ \\ ${ }^{1}$ School of Mechanical Engineering, Southeast University, 210096Nanjing, China \\ ${ }^{2}$ School of Instrument Science and Engineering, Southeast University, 210096Nanjing, China
}

\begin{abstract}
At present, the domestic production of the winding workpiece automatically has been achieved, but the workpiece inspection is mainly relied on human eyes. As the workers is easy to be fatigue, which often causing error checking. In order to improve the efficiency of product inspection and found the problem timely to avoid producing a large number of defective workpieces, the demand of automatic detection winding workpiece is need to be solved eagerly. Take the dc motor rotor for example, according to the characteristics of the rotor, the methodof template matching is been used to locate the workpiece, and the method of Hu moments similarity is used to judge workpiece is qualified or not.The experimental results shows that these method is efficient and effective.
\end{abstract}

Keywords: thresholding; template matching; hu moments.

\section{Introduction}

At present, digital image processing technology is gradually applied in the inspection of industrial workpiece, which improving the efficiency of production and that make the image processing more and more important in automatic identification. However, most of the workpiece of qualified testing link is still stay in artificial detection. For example, though the rotor of electrical machine has been produced automatically and its annual output up to hundreds of millions of sets, but it is still detected by artificiality. What's more, as the rotor commentator need to be around by coil, which is easy prone to leakage or abnormality. As the workers inspect the rotor by eyes, which is easily effect the product qualified rate and production efficiency. Therefore automatically identify coil workpieceis in urgent need to be solved.

Momentsinvariant as the target identification is an important method in image processing, which has been into people's focus and favor. Among them, the Hu moments of identification[1-3] characteristics of image have translation, rotation and scale invariance characteristics, which is the main method of image identification. Therefore the moment through extracting the characteristics of the workpiece, provides a lot of convenience in the workpiece classification and identification.

According to its own characteristics of the workpiece, the workpiece with permanent position of fault area and the correct shape is oneness. The article adopts the methods of template position and template identification for related research.

\footnotetext{
${ }^{\text {a } C o r r e s p o n d i n g ~ a u t h o r ~: ~ z x g 519 @ s i n a . c o m ~}$
} 


\section{Template matching positioning}

In order to prevent the size of workpiecesimages are toodifferent, we fixed the relative position of the workpiece and the camera, so as to avoid losing information of the images.

Template matching [4] is a technology that looking for the most similar part in an image with the template image. Template matching's main content is moving the template image TEMP (the size of the TEMP is $\mathrm{w}^{*} \mathrm{~h}$ ) in the detected image (the size of the IMAGE is $\mathrm{W}^{*} \mathrm{H}$ ) to make relevant calculation, according to the different image matching algorithm, get the most similar location by maximum or minimum value. Because template TEMP need make relevant calculation in (W$\mathrm{w}+1) *(\mathrm{H}-\mathrm{h}+1)$ parameters, which has large calculations. In order to reduce the computational work, firstly, select the rotor part of image location, then determine the position of the rotor through template matching. Because the brush rotor commutators are all the same, and the main error always occur at the commutator's hook where need twined by coils, so let the hook part as the location template which is showed in Figure2, and the rotor commutator's location is get in Figure3 through template matching.

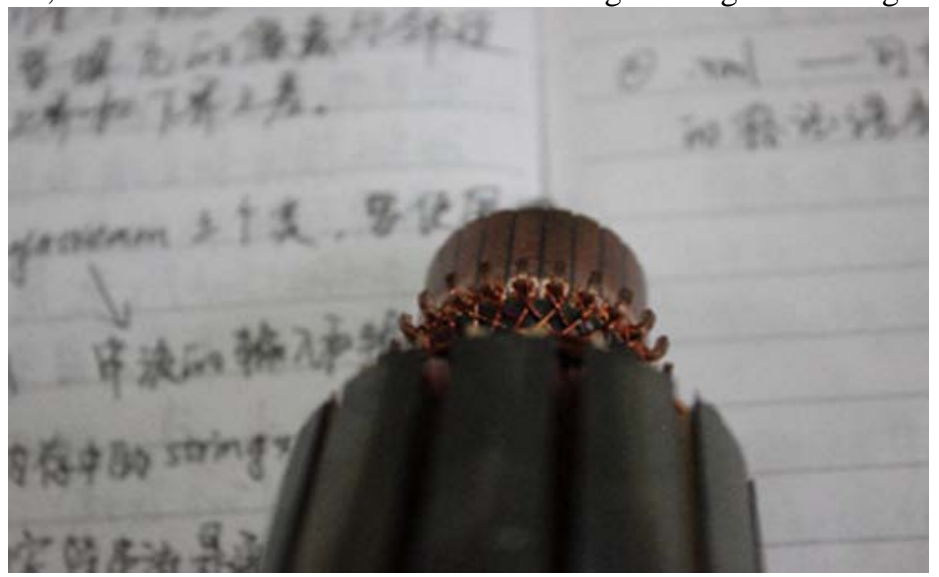

Figure 1. The image to be detected.

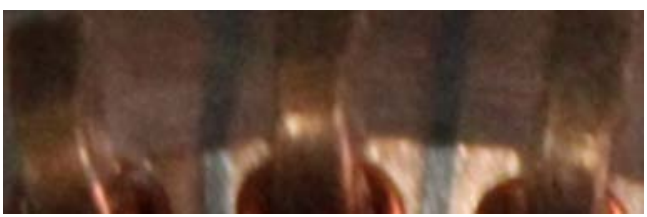

Figure 2. The positioning template.

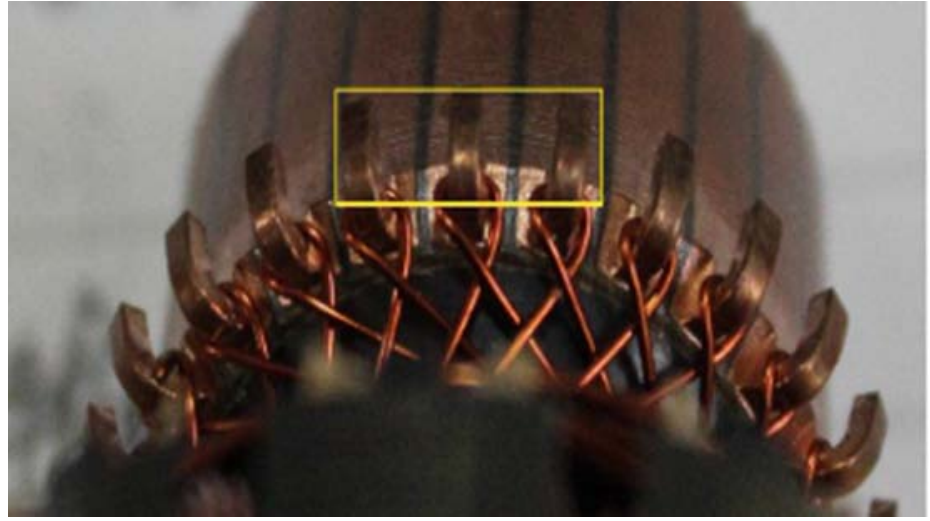

Figure 3. The positioning result. 


\section{Image preprocessing and contour description}

When the rotor has been positioned in the image, the area where need to be tested can be obtained Figure4.1 showing three qualified coils clearly, and the unqualified coils are showed in Figure4.2 and Figure4.3, such as leakage, bolt or deformity. Because there is only one qualified style form and unqualified style variety, so the qualified winding coil can be a template. Extract the Hu moment characteristics of template coil and coil under test, then the coil can be determined is qualified or not by different characteristics between them.

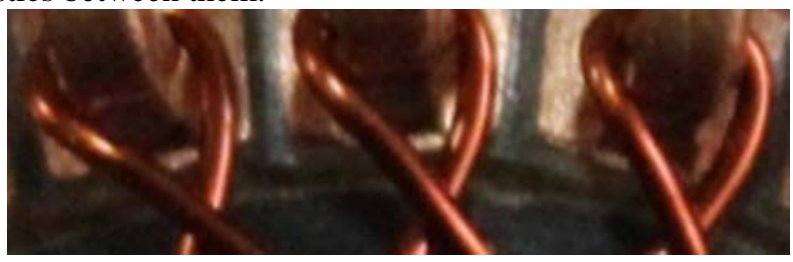

Figure 4.1. The area to be tested.

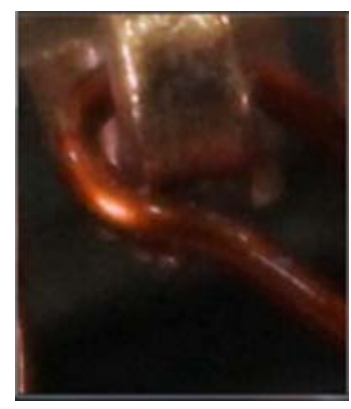

Figure 4.2. Deformity.

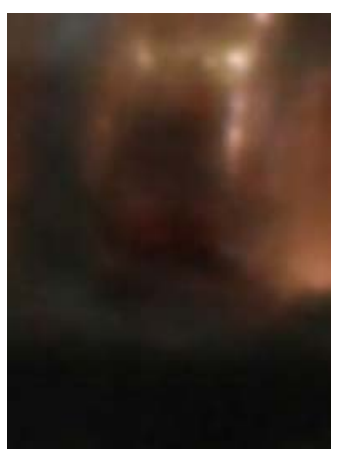

Figure 4.3. Leakage.

Firstly, preprocess the rotor image use filtering de-noising, gray scale interference or binarization and so on. Secondly, get the image's outline feature by adaptive operation, the function findContours() and drawContours() packaged in opencv can drawing the contours, and the results are drawn in Figure5 and Figure6. 


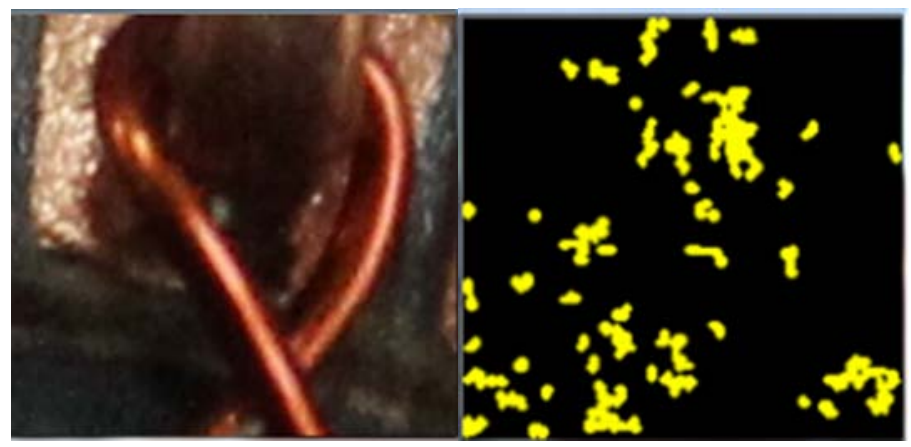

Figure 5. The qualified coil's outline feature.

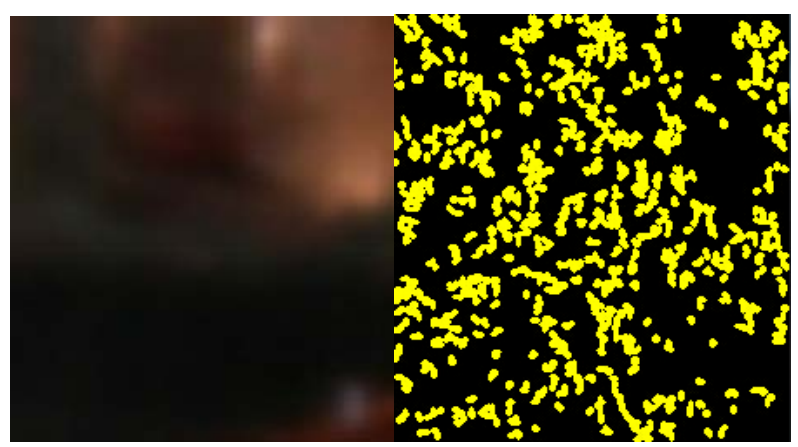

Figure 6. The unqualified coil's outline feature.

Whether the rotor commutator has a coil can be seen from the results obviously, therefore their moments characteristics will be large difference. So, if use the qualified workpiece image's contour feature as a judge, it can be easily to judge whether the workpiece is qualified.

\section{Moments and Hu moments}

Moment is a concept of probability and statistics, is a kind of digital characteristics of random variables. Set for the random variable $\mathrm{x}, \mathrm{c}$ is a constant, $\mathrm{k}$ is a positive integer. The variable E[(xc) $\left.{ }^{\mathrm{k}}\right]$ called $\mathrm{k}$ moments of c point about variable $\mathrm{x}$. In an image, if the pixel coordinates are looked as a twodimensional random variables (X, Y), then a picture of a gray image can be represented using twodimensional gray density function. Therefore moments can be used to describe the characteristic of the gray image.

A picture of $\mathrm{M} * \mathrm{~N}$ digital image, The $\mathrm{p}+\mathrm{q}$ geometric moment center moment, $m_{p q}$ and $\mu_{p q}$ the normalized central moment $\eta_{p q}$ are shown in the below.

$$
\begin{gathered}
m_{p q}=\sum_{i=1}^{M} \sum_{j=1}^{N} i^{p} j^{q} f(i, j) \\
\mu_{p q}=\sum_{i=1}^{M} \sum_{j=1}^{N}(i-\bar{l})^{p}(j-\bar{\jmath})^{q} f(i, j) \\
\eta_{p q}=\frac{\mu_{p q}}{\left(\mu_{00}\right)^{\gamma}}
\end{gathered}
$$

In the equations, $f(i, j)$ is the grey value in the coordinate point (i, $j$ ) of the image. $=\mathrm{m}_{10} / \mathrm{m}_{00},=\mathrm{m}_{01} / \mathrm{m}_{00}, \gamma=\frac{p+q+1}{2}, \mathrm{p}+\mathrm{q}=2,3 \ldots$ The $\mathrm{m}_{00}$ may be regarded as zero moments of the gray level 
image quality, while $\left(\bar{i}_{s} \bar{J}\right)$ describe the image centroid coordinates is one moment, and center moment $\mu_{p q}$ reflects the image relative to the center of mass distribution of grayscale value.

Hu moments[5-7] invariant moment is the linear combination of the above three kinds of moments, it has seven total:

$$
\begin{gathered}
\Phi 1=\eta 20+\eta 02 \\
\Phi 2=(\eta 20-\eta 02) 2+4 \eta 211 \\
\Phi 3=(\eta 20-3 \eta 12) 2+3(\eta 21-\eta 03) 2 \\
\Phi 4=(\eta 30+\eta 12) 2+(\eta 21+\eta 03) 2 \\
\Phi 5=(\eta 30+3 \eta 12)(\eta 30+\eta 12)[(\eta 30+\eta 12) 2-3(\eta 21+\eta 03) 2]+(3 \eta 21-\eta 03)(\eta 21+\eta 03)[3(\eta 30+\eta 12) 2-(\eta 21 \\
+\eta 03) 2] \\
\Phi 6=(\eta 20-\eta 02)[(\eta 30+\eta 12) 2-(\eta 21+\eta 03) 2]+4 \eta 11(\eta 30+\eta 12)(\eta 21+\eta 03) \\
\Phi 7=(3 \eta 21-\eta 03)(\eta 30+\eta 12)[(\eta 30+\eta 12) 2-3(\eta 21+\eta 03) 2]+]+(3 \eta 12-\eta 30)(\eta 21+\eta 03)[3(\eta 30+\eta 12) 2-(\eta \\
21+\eta 03) 2]
\end{gathered}
$$

Image moment characteristics are based on various moments to describe the statistical characteristics of image gray, its characteristic is not affected by the translation, rotation, scale change. So the moment function in binary images and grayscale images has been widely applied. Hu moment function is also a global invariant, it is less sensitive to noise. Whether the target is closed or not, can be identified very well.

\section{The feature extraction and identification of Hu moments}

This paper takes qualified coil workpiece as the template image for identification. Compare the characteristics of template image and the testing image, according to their difference the result will be ensured about the rotor is qualified or not.

When the Hu moments characteristics of the template coil image and the under test coil image are gotten respectively, the $\mathrm{Hu}$ moments characteristics can compose a three dimensional matrix and describe their image separately. huVec $=[\mathrm{v} 1, \mathrm{v} 2, \mathrm{v} 3], \mathrm{v} 1=\Phi 4 / \Phi 3, \mathrm{v} 2=\Phi 7 / \Phi 5, \mathrm{v} 3=\Phi 5 /(\Phi 3 * \Phi 4)$. Then get the similarity of characteristic matrix, set the appropriate threshold, the coil workpiece whether qualified can be telled. Double Xs $=0$; double Xt = 0; double Xst = 0, double $S=X s t / s q r t(X s * X t)$.

\section{The experiment and conclusion}

Now select eight good and 8 bad coil image as a sample to be detected, and choose two qualified coil images as a template. The similarity of the coil image and the template image is shown in the following Table 1.

Table 1. The list of similarity of data.

\begin{tabular}{|c|c|c|c|c|c|c|c|c|}
\hline GOOD COIL & 1 & 2 & 3 & 4 & 5 & 6 & 7 & 8 \\
\hline SIMILAR-ITY & 0.974 & 0.912 & 0.941 & 0.943 & 0.855 & 0.91 & 0.940 & 0.964 \\
\hline $\begin{array}{c}\text { BAD } \\
\text { COKL }\end{array}$ & 1 & 2 & 3 & 4 & 5 & 6 & 7 & 8 \\
\hline SIMILAR-ITY & 0.065 & 0.612 & 0.583 & 0.245 & 0.534 & 0.762 & 0.885 & 0.528 \\
\hline
\end{tabular}


From the experimental results the characteristics of the image is obvious. The similarity of the qualified coil images and the template images is very high, most of them is more than 0.900 , and the similarity of the most unqualified coil images and the template images is less than 0.800 . The similarity of the gap of the unqualified coil image with the template image is changed with the deformation degree, but the difference is larger from the whole result. According to the results of similarity, chose 0.900 as the judging threshold, the accuracy is $93.8 \%$, all of the defective items were found but there is one qualified workpiece considered to be unqualified. Error rate of $6.25 \%$, and Real defective goods accounted for $88.9 \%$ of all defective goods, which is meet the testing requirements.

\section{Acknowledgment}

The authors acknowledge the support of The National Key Technologies R\&D Program of China during the 12th Five-Year Period (No.2013BAJ13B01).

\section{References}

1. AJian Li Qiong. Character recognition based on Hu moment and Zernike moment, (2009)

2. Yao Lei, Based on Hough transform and invariant moment of image pattern recognition technology research, (2006)

3. Wu Chao Yang, Machine vision int the image processing method in the detection of workpiece, (2014)

4. Chen Wen Kai, Zhang Hong Based on wavelet transform and invariant moment of image target recognition algorithm research, (2004)

5. Han Yu Bing, Long Rui Lin, Based on the image analysis of Krawtchouk moments, (1994)

6. Wang Xue and Xiao Bin, Image geometric transformation invariance recognition based on invariant moments, (2007)

7. He Neng Tong and Li Rong Jia, Winding defects detection based on machine vision technology research and application, (2013) 